\title{
Optimal staging system for predicting the prognosis of patients with hepatocellular carcinoma in China: a retrospective study
}

\author{
Lihui Su ${ }^{1 \dagger}$, Tao Zhou ${ }^{1 \dagger}$, Zongli Zhang ${ }^{2}$, Xiuguo Zhang ${ }^{2}$, Xuting Zhi ${ }^{2}$, Caixia Li ${ }^{3}$, Qingliang Wang ${ }^{3}$, Chongqi Jia ${ }^{4}$,
} Wenna Shi ${ }^{1}$, Yanqiu Yue ${ }^{1}$, Yanjing Gao ${ }^{1 *}$ and Baoquan Cheng ${ }^{1 *}$

\begin{abstract}
Background: Several staging systems have been developed to evaluate patients with hepatocellular carcinoma (HCC), including the China Staging System (CS), the American Joint Committee on Cancer (AJCC) tumor-node-metastasis (TNM) staging system, and seventh edition; the Barcelona Clinic Liver Cancer (BCLC) staging system, and Cancer of the Liver Italian Program (CLIP) staging system. The optimal staging system for to evaluate patients in China with HCC has not been determined. This study was designed to determine the optimal staging system for predicting patient prognosis by comparing the performances of these four staging systems in a cohort of Chinese patients with HCC.

Methods: This study enrolled 307 consecutive Chinese patients with HCC in Shandong Province. The performances of the CS, TNM, BCLC, and CLIP staging systems were compared and ranked using a concordance index. Predictors of survival were identified using univariate and multivariate Cox model analyses.

Results: The mean overall survival of the patient cohort was $12.08 \pm 11.87$ months. Independent predictors of survival included tumor size, number of lesions, tumor thromboses, cirrhosis, serum albumin level and serum total bilirubin level. Compared with the other three staging systems, the CS staging system showed optimal performance as an independent predictor of patient survival. The BCLC staging system showed the poorest performance; its treatment algorithm was not suitable for patients in this study.
\end{abstract}

Conclusions: CS was the most suitable staging system for predicting survival of patients with HCC in China.

Keywords: Hepatocellular carcinoma, Prognosis, Staging system, Independent predictors, Overall survival

\section{Background}

Hepatocellular carcinoma (HCC) is the sixth most common cancer and the third leading cause of cancer deaths worldwide [1]. Approximately $55 \%$ of patients with HCC live in China and the 5-year overall survival (OS) rate is only $7 \%$ [2]. Unlike other solid tumors, the prognosis and treatment options for patients with HCC depend not only on the tumor stage but also on residual liver function [3]. Many staging systems that include both the liver cancer and residual liver function have been developed, including the Cancer of the Liver Italian Program (CLIP); the Barcelona Clinic Liver Cancer (BCLC), the American Joint

\footnotetext{
*Correspondence: yanjinggao@aliyun.com; drcbq@163.com

${ }^{\dagger}$ Equal contributors

'Department of Gastroenterology, Qilu Hospital, School of Medicine,

Shandong University, Jinan 250012, China

Full list of author information is available at the end of the article
}

Committee on Cancer (AJCC) tumor-node-metastasis (TNM), seventh edition and the China Staging (CS) systems [4-8].

Many clinical trials in western countries have evaluated the staging, natural history and prognosis of patients with HCC, with highly variable $[9,10]$. Despite China having a greater disease burden than the rest of the world, few studies have been performed in China. Shandong Province, located in the east of China, has a high incidence of HCC. To date, the tumor staging system optimal for evaluating patients with HCC in Shandong province has not been determined. This retrospective study compared the performances of four staging systems, the CLIP, BCLC, AJCC TNM 7th edition, and CS staging systems, in patients with HCC in Shandong Province, China, who were treated at Qilu Hospital of Shandong University. This study also 
attempted to identify factors independently prognostic of survival in these patients.

\section{Methods}

This study was approved by the institutional ethical committee at Qilu Hospital of Shandong University. All patients or their family provided written informed consent for their clinical records to be stored in the hospital database and used for research.

\section{Patients}

Between January 1, 2010, and October 31, 2014, 673 consecutive patients diagnosed with liver cancer were seen at Qilu Hospital of Shandong University. Of these, 366 patients were excluded, including 152 lost to follow-up, 88 with missing data, 58 with cancer of other organs or tissues metastasis to the liver, 47 diagnosed with intrahepatic cholangiocellular carcinoma, and 21 diagnosed at other centers and referred to Qilu Hospital. The remaining 307 patients with HCC were consecutively enrolled and retrospectively analyzed (Fig. 1).

Baseline information, including the results of clinical examinations, laboratory evaluations, imaging modalities (e.g. computed tomography [CT], magnetic resonance imaging [MRI] and/or ultrasonography), was collected at the time of diagnosis. OS was defined the time from the date of initial diagnosis of $\mathrm{HCC}$ to the date of death, last follow-up or the date of censoring (January 1, 2015), whichever came first.

HCC diagnosis was confirmed by histopathological examination of surgical samples or cytologic evaluation of needle biopsy samples (especially if mass less than $2 \mathrm{~cm}$ ). Alternatively, a diagnosis of HCC was based on the radiologic criteria of the European Association for the Study of the Liver (EASL) [11, 12]. Based on collected data, all included patients were restaged retrospectively according to the CLIP, BCLC, AJCC TNM seventh edition, and CS staging systems.

\section{Statistical methods}

All patients were followed up until death or January 1, 2015. Continuous variables were expressed as mean \pm standard deviation (SD), and categorical as frequencies and percentage. Survival outcomes were estimated by the Kaplan-Meier method and compared by the log-rank test.

Staging systems were ranked using the concordance index (c-index), which measures the capacity of the different staging systems to stratify patients with different outcomes: the higher the c-index, the more informative the model was about patient outcomes.

Independent prognostic factors were identified through backward stepwise selection in a Cox regression model. Variables significant $(p<0.05)$ on univariate analysis were included in the multivariate Cox proportional hazards model. Adjusted hazard ratios (HRs) and $95 \%$ confidence intervals (95\% CIs) were calculated.

All statistical analyses were performed using STATA/SE version 13.1 software (Stata Corporation, College Station, TX, USA). All $p$-values were two-sided, and those less than 0.05 were considered statistically significant.

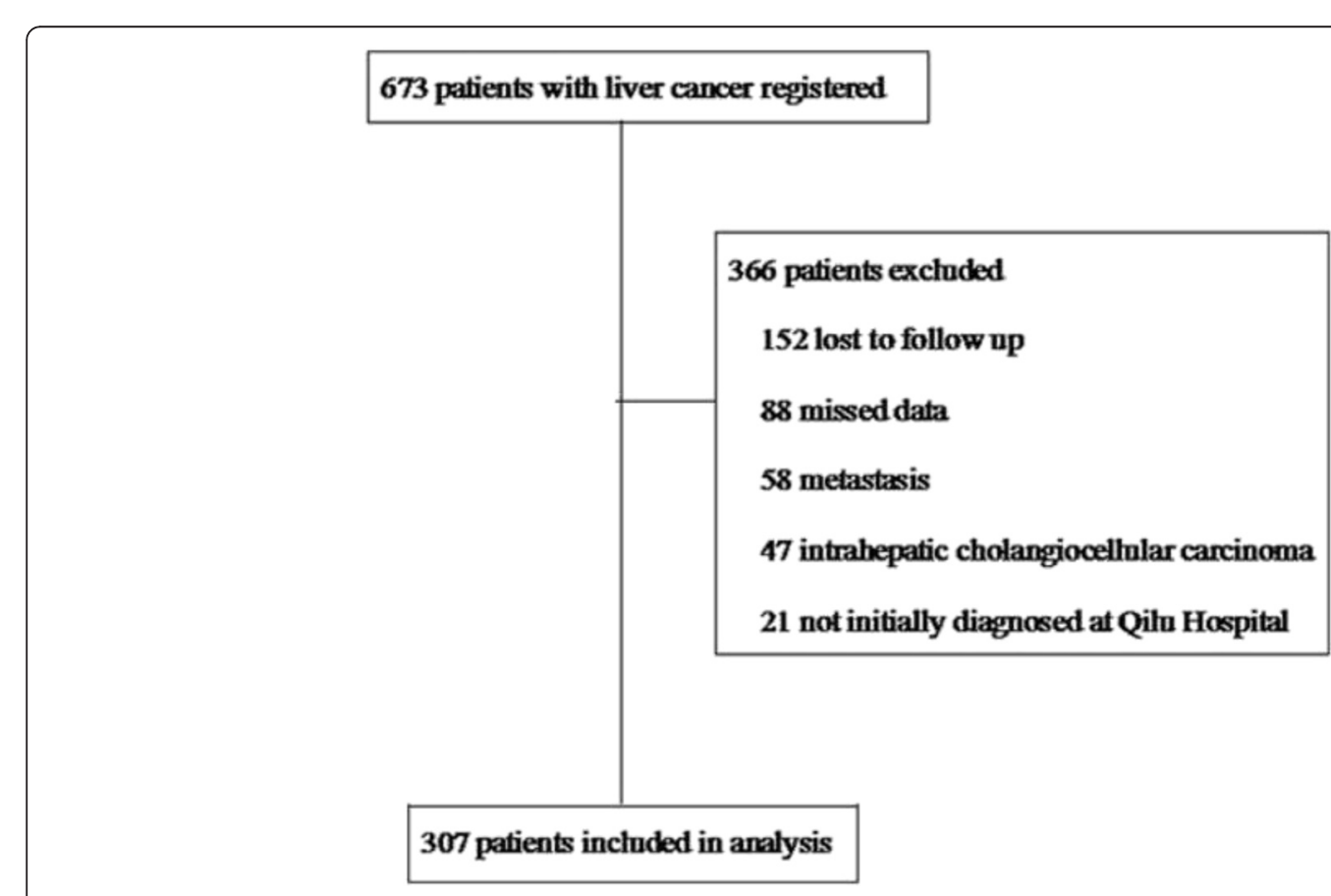

Fig. 1 Flow chart of the study 
Table 1 Baseline demographic and clinical characteristics of the 307 patients with hepatocellular carcinoma (Additional files 1 and 2)

\begin{tabular}{|c|c|}
\hline Characteristic & \\
\hline \multicolumn{2}{|l|}{ Age, years } \\
\hline Mean \pm SD & $55.43 \pm 10$ \\
\hline Range & $11-84$ \\
\hline \multicolumn{2}{|l|}{ Sex, \% } \\
\hline Male & $252(82.1)$ \\
\hline Female & $55(17.9)$ \\
\hline \multicolumn{2}{|l|}{ ECOG PS, \% } \\
\hline 0 & 100 (32.6) \\
\hline 1 & $146(47.6)$ \\
\hline 2 & $44(14.3)$ \\
\hline 3 & $16(5.2)$ \\
\hline 4 & $1(0.3)$ \\
\hline \multicolumn{2}{|l|}{ Etiology, \% } \\
\hline HBV & $239(77.8)$ \\
\hline $\mathrm{HCV}$ & $7(2.3)$ \\
\hline $\mathrm{HBV}+\mathrm{HCV}$ & $2(0.7)$ \\
\hline Alcoholism & $5(1.6)$ \\
\hline Other & $54(17.6)$ \\
\hline \multicolumn{2}{|c|}{ Child-Pugh Grade, \% } \\
\hline A & $239(77.9)$ \\
\hline B & $56(18.2)$ \\
\hline C & $12(3.9)$ \\
\hline \multicolumn{2}{|c|}{ Child-Pugh Score, \% } \\
\hline 5 & $176(57.4)$ \\
\hline 6 & $63(20.5)$ \\
\hline 7 & $36(11.7)$ \\
\hline 8 & $9(2.9)$ \\
\hline 9 & $11(3.6)$ \\
\hline 10 & $9(2.9)$ \\
\hline 11 & $3(1.0)$ \\
\hline
\end{tabular}

Hepatic encephalopathy, \%

No

Yes

Ascites, \%

No

Little

Middle

Large

Portal hypertension, \%

No

Yes

Cirrhosis, \%

No

Yes
Table 1 Baseline demographic and clinical characteristics of the 307 patients with hepatocellular carcinoma (Additional files 1 and 2) (Continued)

\begin{tabular}{|c|c|}
\hline \multicolumn{2}{|l|}{ Laboratory values, mean \pm SD } \\
\hline Total bilirubin $(\mu \mathrm{mol} / \mathrm{l})$ & $24.55 \pm 50.93$ \\
\hline Albumin $(g / l)$ & $38.53 \pm 5.78$ \\
\hline Prothrombin time (sec) & $0.19 \pm 0.80$ \\
\hline \multicolumn{2}{|l|}{$\operatorname{AFP}(\mathrm{ng} / \mathrm{ml}), \%$} \\
\hline$<400$ & $211(68.7)$ \\
\hline$\geq 400$ & $96(31.3)$ \\
\hline \multicolumn{2}{|l|}{ Tumor characteristics } \\
\hline Tumor size (mean $\pm S D, c m)$ & $6.18 \pm 4.04$ \\
\hline \multicolumn{2}{|l|}{ Number of lesions, \% } \\
\hline 1 & $200(65.2)$ \\
\hline $2-3$ & $29(9.4)$ \\
\hline$\geq 4$ & $78(25.4)$ \\
\hline \multicolumn{2}{|l|}{ Lobar involvement, \% } \\
\hline Unilobar & $164(53.4)$ \\
\hline$\geq$ bilobar & $143(46.6)$ \\
\hline \multicolumn{2}{|l|}{ Tumor morphology, \% } \\
\hline Uninodular & $147(47.9)$ \\
\hline Multinodular & $77(25.1)$ \\
\hline Massive, diffuse & $83(27.0)$ \\
\hline \multicolumn{2}{|l|}{ Vascular and/or organ invasion, \% } \\
\hline No & $235(76.6)$ \\
\hline Portal/hepatic vein & $52(16.9)$ \\
\hline Other vascular & $9(2.9)$ \\
\hline Organ invasion & $11(3.6)$ \\
\hline \multicolumn{2}{|l|}{$N, \%$} \\
\hline No & $252(82.1)$ \\
\hline Yes & $55(17.9)$ \\
\hline \multicolumn{2}{|l|}{$M, \%$} \\
\hline No & $278(90.6)$ \\
\hline Yes & $29(9.4)$ \\
\hline \multicolumn{2}{|l|}{ Tumor thrombosis, \% } \\
\hline None & $247(80.4)$ \\
\hline Portal stem vein & $26(8.5)$ \\
\hline Inferior vena cava & $6(1.9)$ \\
\hline Hepatic vein branches & $2(0.7)$ \\
\hline Portal vein branches & $16(5.2)$ \\
\hline Vessel & $3(1.0)$ \\
\hline Hepatic duct & $2(0.7)$ \\
\hline $\begin{array}{l}\text { Inferior vena cava branches and } \\
\text { Portal vein branches and/or } \\
\text { Hepatic vein branches }\end{array}$ & $5(1.6)$ \\
\hline \multicolumn{2}{|l|}{ Current outcomes, \% } \\
\hline Dead & $120(39.1)$ \\
\hline Alive & $187(60.9)$ \\
\hline $\mathrm{OS}$, mean $\pm \mathrm{SD}$, months & $12.08 \pm 11.87$ \\
\hline
\end{tabular}




\section{Results}

\section{Patient characteristics}

Of the 307 patients with HCC included in the study, 252 (82.1\%) were male and 55 (17.9\%) were female, with a mean age of $55.43 \pm 10.69$ years. Among the 307 patients with HCC, 143 (46.6 \%) were confirmed by histopathological examination of surgical samples or cytologic evaluation of needle biopsy samples, that the main tumor differentiation was intermediate (40.6\%), and 164 (53.4\%) were diagnosed by the imaging criteria. The most frequent etiology of underlying liver disease was hepatitis B virus (HBV) infection (77.8\%), followed by other etiology (17.6\%), hepatitis $\mathrm{C}$ virus ( $\mathrm{HCV}$ ) infection $(2.3 \%)$ and alcoholism (1.6\%); only $0.7 \%$ of patients were infected with

Table 2 Tumor staging information of the 307 patients with hepatocellular carcinoma

\begin{tabular}{|c|c|}
\hline Staging system & Patients (\%) \\
\hline \multicolumn{2}{|l|}{ CLIP } \\
\hline 0 & $95(30.9)$ \\
\hline 1 & $86(28.0)$ \\
\hline 2 & $42(13.7)$ \\
\hline 3 & $53(17.3)$ \\
\hline 4 & $21(6.8)$ \\
\hline 5 & $8(2.6)$ \\
\hline 6 & $2(0.7)$ \\
\hline \multicolumn{2}{|l|}{$\mathrm{BCLC}$} \\
\hline 0 & $10(3.3)$ \\
\hline A & $45(14.7)$ \\
\hline B & $32(10.4)$ \\
\hline C & $196(63.8)$ \\
\hline D & $24(7.8)$ \\
\hline \multicolumn{2}{|l|}{ TNM $^{\mathrm{a}}$} \\
\hline । & $133(43.3)$ \\
\hline$\|$ & $41(13.4)$ \\
\hline$\| \mathrm{A}$ & $15(4.9)$ \\
\hline$\| \mathrm{II}$ & $37(12.0)$ \\
\hline$\| I I C$ & $11(3.6)$ \\
\hline IVA & $41(13.4)$ \\
\hline IVB & $29(9.4)$ \\
\hline \multicolumn{2}{|l|}{ CS } \\
\hline la & 49 (16.0) \\
\hline $\mathrm{lb}$ & $42(13.7)$ \\
\hline$\| \mathrm{a}$ & $51(16.6)$ \\
\hline $11 \mathrm{~b}$ & $63(20.5)$ \\
\hline Illa & $90(29.3)$ \\
\hline IIlb & $12(3.9)$ \\
\hline
\end{tabular}

Table 3 Univariate analyses of factors independently prognostic of overall survival in the 307 patients with hepatocellular carcinoma

\begin{tabular}{|c|c|c|c|c|c|}
\hline Variable & Coefficient & SE & $P$ & $H R$ & $95 \% \mathrm{Cl}$ \\
\hline Sex & 0.39 & 0.28 & 0.156 & 1.48 & $0.86-2.54$ \\
\hline Age & -0.09 & 0.08 & 0.293 & 0.92 & $0.78-1.08$ \\
\hline ECOG PS & 0.74 & 0.10 & 0.000 & 2.10 & $1.74-2.54$ \\
\hline Tumor size & 0.15 & 0.02 & 0.000 & 1.16 & $1.12-1.21$ \\
\hline Number of lesions & 0.58 & 0.10 & 0.000 & 1.78 & $1.47-2.17$ \\
\hline Lobar involvement & 1.16 & 0.20 & 0.000 & 3.20 & $2.18-4.70$ \\
\hline Tumor formation & 0.70 & 0.11 & 0.000 & 2.01 & $1.63-2.49$ \\
\hline Ascites & 0.44 & 0.10 & 0.000 & 1.55 & $1.27-1.89$ \\
\hline Total bilirubin & 0.68 & 0.14 & 0.000 & 1.98 & $1.51-2.60$ \\
\hline Albumin & -0.10 & 0.02 & 0.000 & 0.91 & $0.88-0.94$ \\
\hline Child-Pugh Grade & 0.86 & 0.16 & 0.000 & 2.37 & $1.72-3.25$ \\
\hline alpha-fetoprotein & 0.72 & 0.18 & 0.000 & 2.05 & $1.43-2.94$ \\
\hline hepatitis B virus & -0.37 & 0.21 & 0.081 & 0.69 & $0.46-1.05$ \\
\hline hepatitis C virus & -0.55 & 0.59 & 0.347 & 0.58 & $0.18-1.82$ \\
\hline Alcoholism & 0.53 & 0.59 & 0.366 & 1.70 & $0.54-5.36$ \\
\hline Other & 0.42 & 0.23 & 0.066 & 1.53 & $0.97-2.39$ \\
\hline Lymph node metastasis & 0.81 & 0.21 & 0.000 & 2.26 & $1.48-3.43$ \\
\hline Distant metastasis & 1.37 & 0.22 & 0.000 & 3.93 & $2.56-6.05$ \\
\hline Tumor thromboses & 1.31 & 0.20 & 0.000 & 3.71 & $2.50-5.50$ \\
\hline Portal hypertension & 0.30 & 0.22 & 0.173 & 1.35 & $0.88-2.09$ \\
\hline Cirrhosis & -0.80 & 0.18 & 0.000 & 0.45 & $0.31-0.64$ \\
\hline \multicolumn{6}{|l|}{ Vascular/organ invasion } \\
\hline Portal/hepatic vein & 1.31 & 0.21 & 0.000 & 3.72 & $2.47-5.60$ \\
\hline Others & 0.79 & 0.33 & 0.015 & 2.21 & $1.17-4.18$ \\
\hline
\end{tabular}

both HBV and HCV. In total, $65.2 \%$ of patients had a single tumor, with a mean tumor size of $6.18 \pm 4.04 \mathrm{~cm}$; and $77.9 \%$ of patients had underlying Child-Pugh class A liver function. Regarding treatment modalities, $56.1 \%$ of patients underwent curative procedures (LR and RFA), whereas TACE, MWA, systemic treatment and supportive care were administrated to $18.9 \%, 3.9 \%, 4.2 \%, 16.9 \%$ of patients, respectively. Of the 307 patients, 120 (39.1\%) had died by the time of the final analysis (January 1,

Table 4 Multivariate analysis of factors prognostic of overall survival in the 307 patients with hepatocellular carcinoma

\begin{tabular}{lcllll}
\hline Variables & Coefficient & $\mathrm{SE}$ & $\mathrm{P}$ & $\mathrm{HR}$ & $95 \% \mathrm{Cl}$ \\
\hline Cirrhosis & -0.56 & 0.21 & 0.008 & 0.57 & $0.38-0.87$ \\
Tumor size & 0.11 & 0.02 & 0.000 & 1.12 & $1.07-1.17$ \\
Number of lesions & 0.36 & 0.11 & 0.001 & 1.44 & $1.16-1.79$ \\
Total bilirubin & 0.56 & 0.16 & 0.000 & 1.74 & $1.28-2.37$ \\
Albumin & -0.04 & 0.02 & 0.019 & 0.96 & $0.93-0.99$ \\
Tumor thromboses & 0.70 & 0.22 & 0.001 & 2.01 & $1.31-3.09$ \\
\hline
\end{tabular}




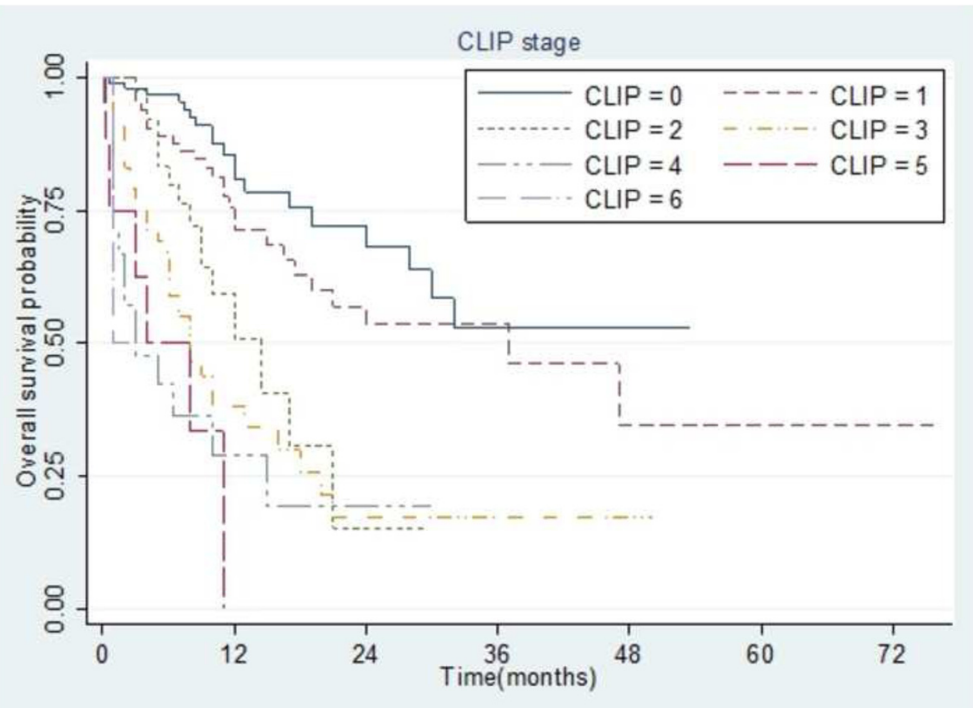

Fig. 2 Kaplan-Meier analysis of overall survival in 307 patients with hepatocellular carcinoma stratified according to the Cancer of the Liver Italian Program (CLIP) staging system. All differences between groups wee statistically significant $(p<0.001)$

2015). The mean OS was $12.08 \pm 11.87$ months (Table 1; Additional files 1 and 2).

Patients were classified into stage groups according to the four staging systems. According to the BCLC staging system, $63.8 \%$ of referred patients had advanced stage tumor stages. In contrast, the different AJCC TNM stages were more evenly distributed, and $96.7 \%$ of patients had CLIP scores $\leq 4$. According to the CS staging system, $29.7,37.1$, and $33.2 \%$ of patients had stages I, II, and III disease, respectively (Table 2).
Baseline predictors of survival

Univariate analysis showed that Child-Pugh grade, tumor size and number, serum total bilirubin and AFP concentrations, tumor thromboses, and cirrhosis were significantly associated with OS (Table 3). Multivariate analysis found that tumor size, number of lesions; serum total bilirubin level and tumor thromboses were the most accurate independent predictors of OS ( $p \leq 0.001$ each). In addition, cirrhosis and albumin were also predictive of reduced OS (Table 4).

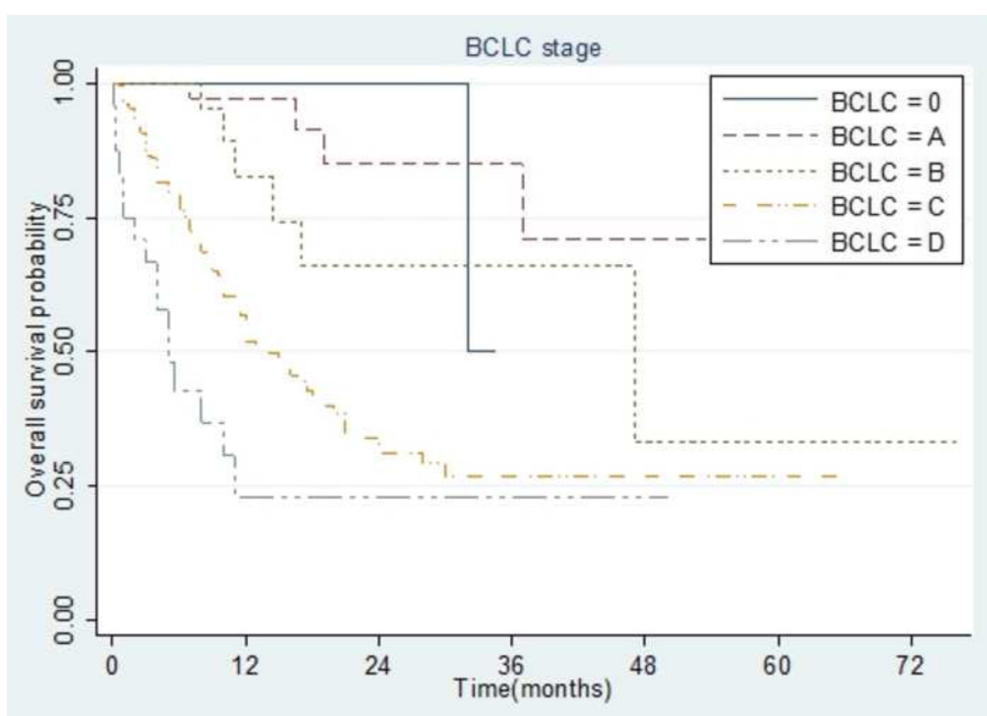

Fig. 3 Kaplan-Meier analysis of overall survival in 307 patients with hepatocellular carcinoma stratified according to the Barcelona Clinic Liver Cancer staging system. All differences between groups were statistically significant $(p<0.001)$ 


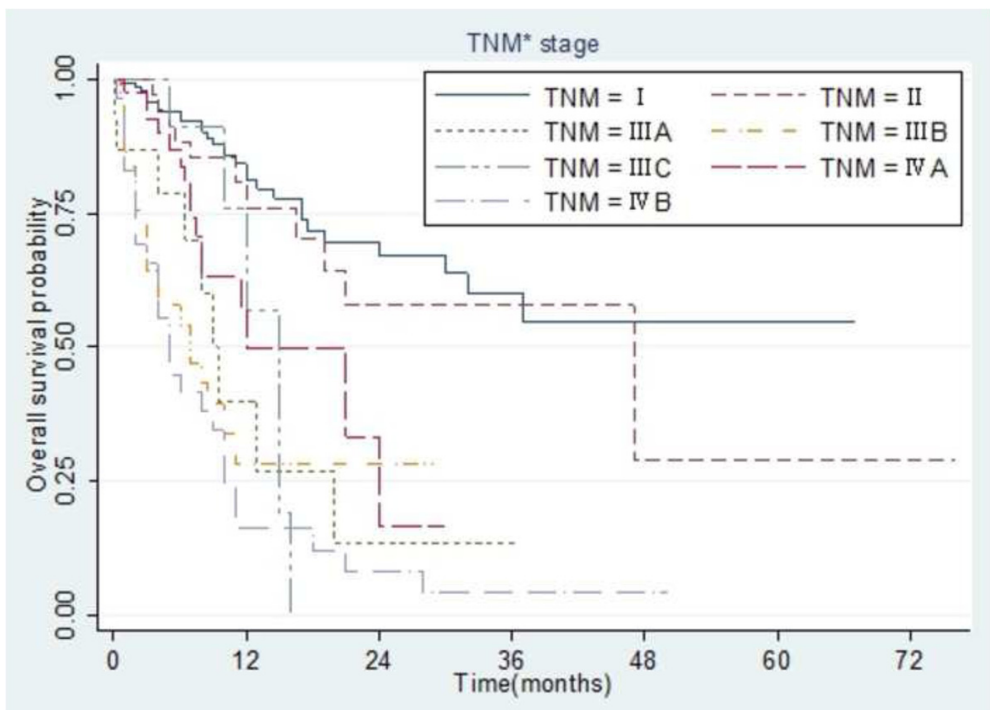

Fig. 4 Kaplan-Meier analysis of overall survival in 307 patients with hepatocellular carcinoma stratified according to the AJCC TNM seventh edition staging system. All differences between groups were statistically significant $(p<0.001)$

\section{Survival comparisons among staging groups}

Survival curves were generated by Kaplan-Meier method for each of the four staging systems. Stage groupings of all four staging systems were significantly predictive of OS ( $p<0.001$ each), although some overlapping of survival curves was observed (Figs. 2, 3, 4 and 5).

\section{Ranking of discriminatory ability of staging system}

The prognostic ability of the different staging systems was compared by calculating the c-index of each. The CS staging system had the highest c-index $(0.75 ; 95 \% \mathrm{CI}$, $0.71-0.80)$, followed by CLIP $(0.74 ; 95 \% \mathrm{CI}, 0.69-0.79)$, the AJCC TNM seventh edition (0.70; $95 \%$ CI, 0.65-0.75), and BCLC $(0.69 ; 95 \%$ CI, 0.65-0.73) staging systems. There was a significant difference between prognostic ability of the CS staging system compared with BCLC staging system $(p=0.031)$. However, it was no statistically difference among the others (CS compared with CLIP, $p=0.130$; CS compared with the AJCC TNM seventh edition, $p=0.746$; CLIP compared with the AJCC

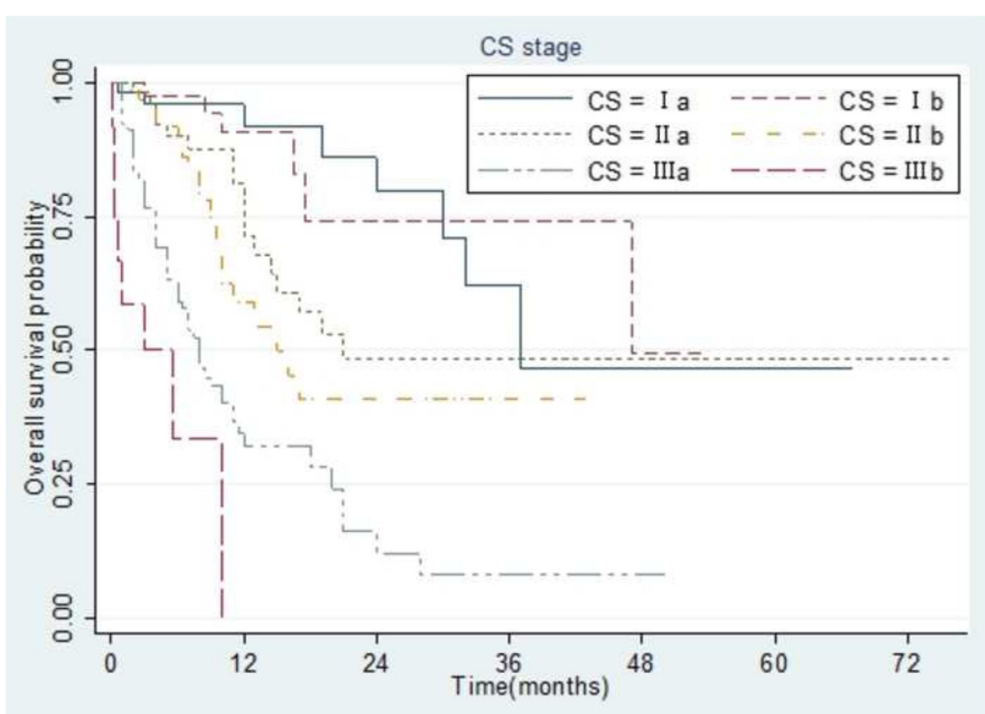

Fig. 5 Kaplan-Meier analysis of overall survival in 307 patients with hepatocellular carcinoma stratified according to the Chinese staging system. All differences between groups were statistically significant $(p<0.001)$ 
Table 5 Ranking of staging systems by concordance indices in patients with hepatocellular carcinoma

\begin{tabular}{llcl}
\hline Rank & System & C-index & $95 \% \mathrm{Cl}$ \\
\hline 1 & CS & 0.75 & $0.71-0.80$ \\
2 & CLIP & 0.74 & $0.69-0.79$ \\
3 & TNM $^{a}$ & 0.70 & $0.65-0.75$ \\
4 & BCLC & 0.69 & $0.65-0.73$ \\
\hline${ }^{\text {a Seventh edition }}$ & & &
\end{tabular}

TNM seventh edition, $p=0.243$; CLIP compared with BCLC, $p=0.661$; the AJCC TNM seventh edition compared with BCLC, $p=0.080$ ) (Table 5).

\section{Discussion}

The predominant etiology of HCC in patients in Shandong Province China was HBV infection. This study of factors independently prognostic of OS in this population found that tumor extent (e.g. tumor size, number of liver lesions, and tumor thromboses), hepatic function (serum total bilirubin concentration and serum albumin level), cirrhosis were independent baseline predictors of OS. Of this patient population, $68.4 \%$ had underlying cirrhosis, which was strongly associated with OS, and 70-80 \% showed histological evidence of liver cirrhosis. AFP was again of limited use in this study, because it was proven to be both not sensitive enough to identify early stage HCC and not specific enough to avoid unnecessary recall procedures, so AFP test has been dropped from the latest Western guideline for the clinical diagnosis of HCC [11-13]. We also founded that serum total bilirubin concentration, serum albumin level and greater tumor extent were related to poor prognosis variables, indicating that the long-term survival of patients with HCC was associated not only with the tumor but with liver function [3, 14-16].

CS was a new staging system proposed by Chinese Society of Liver Cancer (CSLC) for the patients with hepatocellular carcinoma and was initially launched in 2001. The CS staging system combined hepatic function, as defined by Child-Pugh classification, and tumor extent, as defined by adjusted TNM stage, that the parameters included tumor size and tumor location, thrombosis (portal vein, inferior vena cava and biliary duct), lymph node metastasis, distant metastasis and the Child-Pugh classification [8]. It classified stages of disease into six subgroups, from Ia to IIIb (Table 6). This study found that the CS staging system had the highest c-index and there was a significant difference between prognostic ability of the CS staging system compared with BCLC staging system $(p=0.031)$. So, the CS staging system was optimal in distinguishing survival categories in patients with HCC in Shandong Province, China. The CS staging system was the most prognostic in our cohort because it included the independent predictors of survival we had identified. These included serum concentration of total bilirubin and serum albumin level, parameters of Child-Pugh grade, which can reflect the residual hepatic function of the patients with HCC; and tumor stage (tumor size, portal vein thromboses, and number of liver lesions). In contrast, the BCLC staging system showed the poorest performance, despite its having been viewed as the standard classification that is used for trial design and clinical management of patients with HCC [17]. Several reasons may explain the unsuitability of the BCLC staging system for Chinese patients with HCC. First, studies have shown that the performance of the BCLC staging system may be better in patients with early than late stage disease $[18,19]$. However, $63.8 \%$ of the patients in our study had advanced stage disease (BCLC stage C), limiting the discriminatory ability of BCLC staging. Second, the natural history of HCC may vary by underlying etiology. The primary cause of HCC in western countries is $\mathrm{HCV}$ infection, whereas the primary cause of $\mathrm{HCC}$ in our population was HBV infection (77.8\%). Therefore, the ability of BCLC staging to stratify Asian patients with HBV-associated HCC remains unclear $[19,20]$.

This study had several potential limitations. First, it was retrospective in design. Moreover, 152 patients were lost to follow up and data were missing for 88 . However,

Table 6 The classification criteria of China staging system

\begin{tabular}{|c|c|c|c|c|c|}
\hline Stage & Tumor size $(\mathrm{cm})$ and location & Thrombosis & $\mathrm{N}$ & M & Child-Pugh score \\
\hline la & single, $\leq 3$ & absent & absent & absent & A \\
\hline $\mathrm{lb}$ & unilobar, $\leq 5$ & absent & absent & absent & A \\
\hline Ila & unilobar, $\leq 10$; or bilobar, $\leq 5$ & absent & absent & absent & A \\
\hline \multirow[t]{2}{*}{ Ilb } & \multirow[t]{2}{*}{ unilobar, >10; or bilobar, > 5; any } & \multirow{2}{*}{$\begin{array}{l}\text { absent; portal vein, or inferior } \\
\text { vena cava, or biliary duct branches }\end{array}$} & absent & absent & \\
\hline & & & absent & absent & $A$ or $B$ \\
\hline \multirow[t]{3}{*}{ Illa } & any; & $\begin{array}{l}\text { portal vein, or inferior vena cava, } \\
\text { or biliary duct stem; }\end{array}$ & any & any & $A$ or $B$ \\
\hline & any; & any; & present & any & \\
\hline & any & any & any & present & \\
\hline IIlb & any & any & any & any & C \\
\hline
\end{tabular}

$N$ lymph node metastasis, $M$ distant metastasis 
many Chinese people live in the countryside, making communication difficult. Thus, there may have been potential bias in patient selection. Secondly, this was a singlecenter study involving patients admitted consecutively to Qilu Hospital of Shandong University for treatment. However, our study had several strengths. Complete data were obtained from a large number of patients. Moreover, the follow-up period was relatively long, and the epidemiological characteristics of our cohort were consistent with those reported in other studies of Chinese patients with HCC [20, 21].

\section{Conclusions}

Of the four HCC staging systems evaluated, the CS staging system was the most informative in predicting survival for patients with HCC in Shandong Province. The poor performance of the BCLC staging system in this cohort suggests its unsuitability for evaluating Chinese patients with HCC. We also found that tumor size, number of lesions, tumor thromboses, serum total bilirubin level; albumin and cirrhosis were the accurate independent predictors of OS.

\begin{abstract}
Abbreviations
$95 \%$ Cl, 95 \% confidence interval; AFP, alpha-fetoprotein; AJCC, American Joint Committee on Cancer; BCLC, Barcelona Clinic Liver Cancer; C-index, concordance index; CLIP, Cancer of the Liver Italian Program; CS, China Staging System; CSLC, Chinese Society of Liver Cancer; CT, computed tomography; ECOG PS, Eastern Cooperative Oncology Group performance status; HBV, hepatitis B virus; HCC, hepatocellular carcinoma; HCV, hepatitis C virus; HR, hazard ratio; $\mathrm{M}$, Distant metastasis; $\mathrm{MRI}$, magnetic resonance imaging; $\mathrm{N}$, Lymph node metastasis; OS, overall survival; SD, standard deviation; SE, standard error; TNM, tumor-node-metastasis
\end{abstract}

\section{Additional files}

Additional file 1: Availability of data and material(1). (XLS 99 kb) Additional file 2: Availability of data and material(2). (DOC $30 \mathrm{~kb}$ )

\section{Acknowledgments}

The authors thank the staff of Qilu Hospital of Shandong University for help with the clinical data. We thank nurses Min Zhang, Aifang Zhu, and Jianrong Bai for their contributions to care and referral of patients as well as data acquisition.

\section{Funding}

Tackling key problems in science and technology of Shandong province and the Fundamental Research Funds of Shandong University (Number: 2009GG20002039; 2014QLKY10) supported this research in the design of the study and collection, analysis, and interpretation of data and in writing the manuscript.

\section{Availability of data and materials}

All datasets on which the conclusions of the manuscript rely to be presented in additional supporting files in excel table format.

\section{Authors' contributions}

BQC, YJG, TZ and LHS conceived the trial concept and designed the protocol. LHS acquired data. CQJ is responsible for analysis and interpretation of data. LHS, BQC, ZT, WNS, and YQY are responsible for drafting of the manuscript. YJG, TZ, LHS, XGZ, XTZ, ZLZ, CXL, QLW, WNS and YQY are responsible for clinical work, and administrative and technical support. BQC is the principle investigator and responsible for trial conduct and critical revisions of the manuscript. All authors aided in drafting the manuscript. All authors have read and approved the final manuscript.

\section{Competing interests}

The authors declare that they have no competing interests.

\section{Consent for publication}

All patients or their family provided written informed consent for their clinical records to be stored in the hospital database and used for research, and consent to publish.

\section{Ethics approval and consent to participate}

This study was approved by the institutional ethical committee at Qilu Hospital of Shandong University (Number: 2014042).

\section{Author details}

${ }^{1}$ Department of Gastroenterology, Qilu Hospital, School of Medicine, Shandong University, Jinan 250012, China. ${ }^{2}$ Department of Hepatobiliary Surgery, Qilu Hospital, School of Medicine, Shandong University, Jinan 250012, China. ${ }^{3}$ Department of Intervention, Qilu Hospital, School of Medicine, Shandong University, Jinan 250012, China. ${ }^{4}$ Department of Epidemiology and Health Statistics, Shandong University, Jinan 250012, China.

Received: 14 December 2015 Accepted: 27 June 2016

Published online: 07 July 2016

\section{References}

1. Forner A, Llovet JM, Bruix J. Hepatocellular carcinoma. Lancet. 2012;379: 1245-55.

2. El-Serag HB, Mason AC. Rising incidence of hepatocellular carcinoma in the United States. N Engl J Med. 1999;340:745-50.

3. Abou-Alfa GK. Hepatocellular carcinoma: Molecular biology and therapy. Semin Oncol. 2006;33:S79-83.

4. A new prognostic system for hepatocellular carcinoma: a retrospective study of 435 patients: the Cancer of the Liver Italian Program (CLIP) investigators. Hepatology.1998;28:751-5.

5. Maida M, Orlando E, Cammà C, Cabibbo G. Staging systems of hepatocellular carcinoma: A review of literature. World J Gastroenterol. 2014; 20:4141-50.

6. Llovet JM, Brú C, Bruix J. Prognosis of hepatocellular carcinoma: the BCLC staging classification. Semin Liver Dis. 1999;19:329-38.

7. Edge SB, Byrd DR, Compton CC, Fritz AG, Greene FL, Trotti A. AJCC cancer staging manual. 7th ed. New York: Springer; 2010.

8. Qu Q, Rui JA, Wang SB, Chen SG, Zhou L, Han K, et al. Comparison of different clinical staging systems for hepatocellular carcinoma. Zhonghua zhong liu za zhi [Chinese journal of oncology]. 2006;28:155-8.

9. Cho YK, Chung JW, Kim JK, Ahn YS, Kim MY, Park YO, et al. Comparison of 7 staging systems for patients with hepatocellular carcinoma undergoing transarterial chemoembolization. Cancer. 2008;112:352-61.

10. op den Winkel M, Nagel D, Sappl J, op den Winkel P, Lamerz R, Zech CJ, et al. Prognosis of patients with hepatocellular carcinoma. Validation and ranking of established staging-systems in a large western HCC-cohort. PLoS ONE. 2012;7:e45066.

11. Bruix J, Sherman M, Llovet JM, Beaugrand M, Lencioni R, Burroughs AK, et al. EASL Panel of Experts on HCC. Clinical management of hepatocellular carcinoma. Conclusions of the Barcelona-2000 EASL conference. European Association for the Study of the Liver. J Hepatol. 2001;35:421-30.

12. Bruix J, Sherman M. Management of Hepatocellular Carcinoma. Hepatology. 2005;42:1208-35.

13. Bruix J, Sherman M. Management of hepatocellular carcinoma: an update. Hepatology. 2011;53:1020-2.

14. Hsu CY, Hsia CY, Huang YH, Su CW, Lin HC, Pai JT, et al. Comparison of surgical resection and transarterial chemoembolization for hepatocellular carcinoma beyond the Milan criteria: a propensity score analysis. Ann Surg Oncol. 2012;19:842-9.

15. Zhou L, Rui JA, Wang SB, Chen SG, Qu Q. Risk factors of poor prognosis and portal vein tumor thrombosis after curative resection of solitary hepatocellular carcinoma. Hepatobiliary Pancreat Dis Int. 2013;12:68-73. 
16. Huitzil-Melendez FD, Capanu M, O'Reilly EM, Duffy A, Gansukh B, Saltz LL, et al. Advanced hepatocellular carcinoma: which staging systems best predict prognosis? J Clin Oncol. 2010;28:2889-95.

17. Llovet JM, Di Bisceglie AM, Bruix J, Kramer BS, Lencioni R, Zhu AX, et al. Panel of Experts in HCC-Design Clinical Trials. Design and endpoints of clinical trials in hepatocellular carcinoma. J Natl Cancer Inst. 2008; 100:698-711.

18. Cillo U, Vitale A, Grigoletto F, Farinati F, Brolese A, Zanus G, et al. Prospective validation of the Barcelona Clinic Liver Cancer staging system. J Hepatol. 2006;44:723-31.

19. Marrero JA, Fontana RJ, Barrat A, Askari F, Conjeevaram HS, Su GL, et al. Prognosis of hepatocellular carcinoma: comparison of 7 staging systems in an American cohort. Hepatology. 2005;41:707-16.

20. Chan SL, Mo FK, Johnson PJ, Liem GS, Chan TC, Poon MC, et al. Prospective validation of the Chinese University Prognostic Index and comparison with other staging systems for hepatocellular carcinoma in an Asian population. J Gastroenterol Hepatol. 2011;26:340-7.

21. Leung TW, Tang AM, Zee B, Lau WY, Lai PB, Leung KL, et al. Construction of the Chinese University Prognostic Index for hepatocellular carcinoma and comparison with the TNM staging system, the Okuda staging system, and the Cancer of the Liver Italian Program staging system: a study based on 926 patients. Cancer. 2002;94:1760-9.

Submit your next manuscript to BioMed Central and we will help you at every step:

- We accept pre-submission inquiries

- Our selector tool helps you to find the most relevant journal

- We provide round the clock customer support

- Convenient online submission

- Thorough peer review

- Inclusion in PubMed and all major indexing services

- Maximum visibility for your research

Submit your manuscript at www.biomedcentral.com/submit
Biomed Central 